\title{
INTERACTIONS OF DELTA SHOCK WAVES FOR THE EQUATIONS OF CONSTANT PRESSURE FLUID DYNAMICS
}

\author{
YU ZHANG* AND YANYAN ZHANG
}

Abstract. The interactions among delta shock waves, vacuum states and contact discontinuities for the equations of constant pressure fluid dynamics are analyzed. By solving the Riemann problem with initial data of three piecewise constant states case by case, the global structures of solutions with four different configurations are constructed. Furthermore, the numerical simulations completely coinciding with theoretical analysis are presented.

Mathematics subject classification (2010): 35L65, 35L67, 76N15.

Keywords and phrases: Constant pressure fluid dynamics, Riemann problem, delta shock wave, vacuum, wave interactions.

\section{REFERENCES}

[1] Y. BRENIER AND E. GRENIER, Sticky particles and scalar conservation laws, SIAM J. Numer. Anal., 35, 6 (1998), 2317-2328.

[2] W. CAI AND Y. ZHANG, Interactions of delta shock waves for zero-pressure gas dynamics with energy conservation law, Advances in Mathematical Physics, 2016, Article ID 1783689 (2016), 12 pages.

[3] H. Cheng, W. LiU And H. YAnG, Two-dimensional Riemann problems for zero-pressure gas dynamics with three constant states, J. Math. Anal. Appl., 343, 1 (2008), 127-140.

[4] R. Courant And K. O. Friedrichs, Supersonic Flow and Shock Waves, Springer-Verlag, New York, 1976.

[5] V. G. Danilov and V. M. Shelkovich, Dynamics of propagation and interaction of $\delta$-shock waves in conservation law systems, J. Differential Equations, 211, 2 (2005), 333-381.

[6] B. T. HAYES AND P. G. LE FLOCH, Measure solutions to a strictly hyperbolic system of conservation laws, Nonlinearity, 9, 6 (1996), 1547-1563.

[7] J. Hu, Two-dimensional Riemann problem for equations of constnat pressure fluid dynamics with functional solutions, Quart. Appl. Math., LVIII, 2 (2000), 251-264.

[8] J. HU, One-dimensional Riemann problem for equations of constant pressure fluid dynamics with measure solutions by viscosity method, Acta Appl. Math., 55, 2 (1999), 209-229.

[9] F. HuAng AND Z. WANG, Well-posedness for pressureless flow, Comm. Math. Phys., 222, 1 (2001), $117-146$.

[10] G. JiAng AND E. TADMOR, Non-oscillatory central schemes for multidimensional hyperbolic conservation laws, SIAM J. Sci. Comput., 19, 6 (1998), 1892-1917.

[11] B. L. Keyfitz AND H. C. KRAnZer, Spaces of weighted measures for conservation laws with singular shock solutions, J. Differential Equations, 118, 2 (1995), 420-451.

[12] S. F. Shandarin AND Y. B. Zeldovich, The large-scale structure of the universe: turbulence, intermittency, structure in a self-gravitating medium, Rev. Mod. Phys., 61, 2 (1989), 185-220.

[13] C. SHEN AND M. Sun, Interactions of delta shock waves for the transport equations with split delta functions, J. Math. Anal. Appl., 351, 2 (2009), 747-755.

[14] W. Sheng AND T. ZHANG, The Riemann problem for transportation equation in gas dynamics, Mem. Am. Math. Soc., 137, 654 (1999), 1-77.

[15] D. TAN AND T. ZhANG, Two-dimensional Riemann problem for a hyperbolic system of nonlinear conservation laws: I. Four-J cases, II. Initial data involving some rarefaction waves, J. Differential Equations, 111, 2 (1994), 203-282. 
[16] D. TAN, T. ZhANG AND Y. ZHENG, Delta shock waves as limits of vanishing viscosity for hyperbolic systems of conservation laws, J. Differential Equations, 112, 1 (1994), 1-32.

[17] E. WeInAN, Yu G. RYKov AND YA G. SinAI, Generalized varinational principles, global weak solutions and behavior with random initial data for systems of conservation laws arising in adhesion particle dynamics, Commun. Math. Phys., 177, 2 (1996), 349-380.

[18] H. YANG, Riemann problems for a class of coupled hyperbolic systems of conservation laws, J. Differential Equations, 159, 2 (1999), 447-484.

[19] H. YANG AND Y. ZHANG, New developments of delta shock waves and its applications in systems of conservation laws, J. Differential Equations, 252, 11 (2012), 5951-5993.

[20] H. Yang AND Y. Zhang, Delta shock waves with Dirac delta function in both components for systems of conservation laws, J. Differential Equations, 257, 12 (2014), 4369-4402.

[21] Q. ZHANG, The vanishing pressure limit of solutions to the simplified Euler equations for isentropic fluids, Annals of Applied Mathematics, 28, 1 (2012), 115-126.

[22] Y. ZHANG AND Y. ZHANG, Generalized plane delta shock waves for the n-dimensional zero-pressure gas dynamics with energy conservation law, Indian J. Pure Appl. Math., 50, 4 (2019), 1067-1086.

[23] Y. ZHANG AND Y. ZHANG, The Riemann problem and interaction of waves in two-dimensional steady zero-pressure adiabatic flow, Int. J. Non-linear Mech., 104, (2018), 100-108. 